\title{
A Comparative Study of ANN and GEP Model to Predict the Pressure Drop in the Water Transportation System
}

\author{
Rajesh Chakraborty, Dr. Uttam Kumar Mandal \\ National Institute of Technology, Agartala, Department of Production Engineering, Tripura, 799046, India \\ Email: rajeshchakra59@gmail.com, uttamkumar_mandal@yahoo.co.in \\ Dr. Rabindra Nath Barman \\ National Institute of Technology, Durgapur, Department of Mechanical Engineering, West Bengal, 713209, India \\ Email: rn.barman@me.nitdgp.ac.in
}

Received: 05 April 2020; Accepted: 24 June 2020; Published: 08 October 2020

\begin{abstract}
In the present study, the parameter responsible to find out pressure drops in a pipeline network system has been modeled by Gene Expression Programming Based on the experimental data. The different factors like Pipe diameter, Particle diameter, liquid density, Solid density liquid Viscosity, Volume fraction, Velocity, Solid concentration are taken into consideration as the input parameter. GEP model was developed to predict the pressure drop within the pipeline system. GEP model predicts the pressure drop with an accuracy of mean R-Square 0.999153373. As the input parameter is responsible for the selection of soft computing method and both ANN and GEP model is considered in order to validate the output parameters. The result of GEP has been compared with an ANN model, to observe the level of accuracy of the predicted pressure drop with a correlation to predict pressure drop shown by equation 6 . The obtained results of both GEP and ANN models are being compared and GEP predicted results are found to be better in predicting the output parameter. The mean absolute error is found to be $15.566 \%$ by the ANN model wherein the GEP model predicts with an accuracy of $8.993 \%$. The results indicate that the GEP is better tool to predict pressure drop with more accuracy.
\end{abstract}

Index Terms: A pressure drop, Multi-phase, Volume fraction, ANN, GEP, solid density, solid concentration, Particle diameter.

\section{Introduction}

A large number of materials as water, oil, the slurry can be transported from place to place by a pipeline network system. Now a day's Pipeline transport has been a progressive technology for transmission a large number of bulk materials. This provides a good profit in terms of cost and saves a lot of time as compared to other types of the transportation system. The main objective of this study is to predict the pressure drop more accurately and to reduce the error percentage and also to develop a correlation to predict pressure drop. The different factors like Pipe diameter, Particle diameter, liquid density, Solid density liquid Viscosity, Volume fraction, Velocity, Solid concentration are taken into consideration as the input parameter. A few numbers of studies are carried out with ANN model but the GEP model is one of the novel approach to predict pressure drop in different pipeline transport system. The characteristics of solids in a liquid flowing through the pipeline network system have been investigated by various researchers for the last 60 years. $[1,2,3,4,5]$ have studied the characteristics of the different parameters of the slurry transport phenomenon. The pressure drop through the pipeline depends upon different flow regimes of the flow characteristics. Many investigations have been made to predict the pressure variation through the pipe [6]. The different layers of the solid particles move with different pressure variation, varied pressure drop. Hence the flow patterns in the outlet changes with a great variation. The flow of solid-liquid concentration in multi-phase flow regimes moving with different velocities and hence the variation in the pressure drop takes place. To propose and develop a pressure drop in a pipeline network system, there is a need to be a correlation that can predict pressure drop over a wide range of working environments. For a commercial system, there needs to be an easy and quick solution to manage the situation. Thus Prediction by ANN and GEP provides a promising solution to predict the pressure drop in a pipeline network system. Artificial neural networks (ANN) are constructed to reproduce processes of the central nervous system of higher creatures. An ANN consists of a set of processing units (nodes) that simulate neurons and are interconnected via a set of "weights" in a way 
that allows signals to travel through the network in parallel. The nodes (neurons) are simple computing elements. They accumulate input from other neurons utilizing a weighted sum. If a certain threshold is reached the neuron passes information to all other linked neurons, otherwise, it remains dormant. Artificial neural networks (ANNs) have extensively used in different engineering application such as adaptive control, model-based control, process monitoring, fault detection, dynamic modeling and parameter estimation in a working environment [7,8] ANN Technique has the wide range of application in the field of Multiphase flow and predicting the holdup and pressure drop variation in a pipeline network analysis. Although, built on the black box architecture the evolved models be short of closed-form analytical relationships between the chosen input and response variables. Based on the sigmoid activation functions, the response within the modeled variables is correlated to the inputs via complex weight matrices which are likely to increase in complexity with the increase in the non-linearity of the problem [9]. An ANN and GEP comparative study has done over unsteady mixed convection over a circular cylinder in the presence of nano-fluid to predict the Average Nusselt Number. The average Nusselt number has found to be proportional to the volume fraction for both the adding and opposing buoyancy conditions. The results obtained by GEP is found to be more efficient in terms of minimum mean relative error [10]. The prediction of aerodynamic forces on a square cylinder propagates through steady and unsteady flow conditions were modeled by back-propagation ANN and GEP tool to determine aerodynamic coefficient, minimum relative error. The results found to be more efficient with the GEP model with a minimum absolute error of 0.00243 percent [11]. ANN as a powerful tool having higher accuracy and efficiency in the flexible fitting of experimental data, prediction, and modeling of flow characteristics modeling. The purpose of the present work is to develop an accurate model based on gene expression programming (GEP) model for prediction of pressure drop in a pipeline network system based on the different flow characteristics. Moreover, the obtained results are compared with the result of ANN to explicitly accurate prediction. The obtained results are found to be more accurate and the superiority of the GEP model over the ANN Model. The data obtained from various kinds of literature are used to analyze both ANN and GEP models is shown in Table1.

\section{Motivation of the Present Study}

Amongst the numerous technical growths approved in defining considered pathways to encounter the pipeline engineering problems, computational methods with its characteristic ability to instantaneously deliver suggestively reduced pipe losses and to describe the flow pattern more accurately in the current research scenario an attempt to predict the pressure drop throughout the pipeline system having a different diameter. The present study tries to find out pressure drop using ANN and GEP was done. This study mainly focuses on the GEP model performance over the ANN model. The GEP model predicts the pressure drop more accurately as compared to ANN model.

\section{Materials and Methods}

The data from various literature have been used to predict the pressure drop in a pipeline with various diameter have been studied by using GEP and ANN model. The results obtained by both the model compared to evaluate the better results. The GEP model predicts more accurately as compared to the ANN model. Experimental analysis in pipe flow and the obtained data [12] as shown in table 1. In this research the experimental data obtained with various pipe diameter are investigated to obtain pressure drop .

Table 1. Data to predict the pressure drop in a pipeline system with different pipe diameter.

\begin{tabular}{ccccccccc}
\hline $\begin{array}{c}\text { Pipe } \\
\text { Diameter(c } \\
\text { m) }\end{array}$ & $\begin{array}{c}\text { Particle } \\
\text { Diameter }\end{array}$ & $\begin{array}{c}\text { Liquid } \\
\text { Density }\end{array}$ & $\begin{array}{c}\text { Solid } \\
\text { Density }\end{array}$ & $\begin{array}{c}\text { Liquid } \\
\text { Viscosity }\end{array}$ & $\begin{array}{c}\text { Volume } \\
\text { Fraction }\end{array}$ & Velocity & $\begin{array}{c}\text { Solid } \\
\text { Concentration }\end{array}$ & $\begin{array}{c}\text { Pressure } \\
\text { Drop }\end{array}$ \\
\hline 5.26 & 38.3 & 1 & 2.33 & 1 & 0.69 & 1.11 & 0.107 & 294.1 \\
5.26 & 38.3 & 1 & 2.33 & 1 & 0.69 & 3.01 & 0.107 & 1651.3 \\
5.26 & 38.3 & 1 & 2.33 & 1 & 0.69 & 4.81 & 0.107 & 3822.9 \\
5.26 & 38.3 & 1 & 2.33 & 1 & 0.69 & 1.33 & 0.306 & 542.9 \\
5.26 & 38.3 & 1 & 2.33 & 1 & 0.69 & 3.12 & 0.306 & 2352.6 \\
5.26 & 38.3 & 1 & 2.33 & 1 & 0.69 & 4.7 & 0.306 & 4727.7 \\
20.85 & 190 & 1 & 1.37 & 1.14 & 0.78 & 2.59 & 0.326 & 266.5 \\
20.85 & 190 & 1 & 1.37 & 1.14 & 0.78 & 2.34 & 0.327 & 226.3 \\
20.85 & 190 & 1 & 1.37 & 1.14 & 0.78 & 2.01 & 0.333 & 177.3 \\
20.85 & 190 & 1 & 1.37 & 1.14 & 0.78 & 1.78 & 0.327 & 147 \\
20.85 & 190 & 1 & 1.37 & 1.14 & 0.78 & 1.59 & 0.323 & 123.4 \\
20.85 & 190 & 1 & 1.37 & 1.14 & 0.78 & 1.37 & 0.327 & 99.9 \\
5.15 & 165 & 1 & 2.65 & 1 & 0.58 & 1.66 & 0.0741 & 666.2 \\
5.15 & 165 & 1 & 2.65 & 1 & 0.58 & 3.78 & 0.0897 & 2449.2 \\
5.15 & 165 & 1 & 2.65 & 1 & 0.58 & 1.66 & 0.1694 & 901.3 \\
\hline
\end{tabular}




\begin{tabular}{ccccccccc}
\hline 5.15 & 165 & 1 & 2.65 & 1 & 0.58 & 4.17 & 0.1886 & 3428.9 \\
5.15 & 165 & 1 & 2.65 & 1 & 0.58 & 1.66 & 0.2669 & 1136.4 \\
5.15 & 165 & 1 & 2.65 & 1 & 0.58 & 4.33 & 0.286 & 4408.1 \\
26.3 & 165 & 1 & 2.65 & 1 & 0.58 & 2.9 & 0.0932 & 261.6 \\
26.3 & 165 & 1 & 2.65 & 1 & 0.58 & 3.5 & 0.0921 & 334.1 \\
26.3 & 165 & 1 & 2.65 & 1 & 0.58 & 2.9 & 0.1759 & 305.7 \\
26.3 & 165 & 1 & 2.65 & 1 & 0.58 & 3.5 & 0.1726 & 382.1 \\
26.3 & 165 & 1 & 2.65 & 1 & 0.58 & 2.9 & 0.2586 & 355.6 \\
26.3 & 165 & 1 & 2.65 & 1 & 0.58 & 3.5 & 0.2597 & 453.6 \\
26.3 & 165 & 1 & 2.65 & 1 & 0.58 & 2.9 & 0.3292 & 414.4 \\
26.3 & 165 & 1 & 2.65 & 1 & 0.58 & 3.5 & 0.3241 & 526.1 \\
49.5 & 165 & 1 & 2.65 & 1 & 0.58 & 3.16 & 0.0943 & 143 \\
49.5 & 165 & 1 & 2.65 & 1 & 0.58 & 3.76 & 0.0923 & 186.1 \\
49.5 & 165 & 1 & 2.65 & 1 & 0.58 & 3.07 & 0.1727 & 157.7 \\
49.5 & 165 & 1 & 2.65 & 1 & 0.58 & 3.76 & 0.1726 & 210.6 \\
\hline
\end{tabular}

\section{A. Analytical Modelling}

In this paper, an analytical study has been carried out by obtaining data from various literature to investigate pressure drop within the flow domain using GEP and

ANN model. The main focus of the present study is to finding pressure drop more accurately over the existing predicted model by various authors.

\section{B. GEP Modelling.}

Gene Expression Programming (GEP) is generally a population-based evolutionary algorithm [13].GEP is a dynamic alternate of Genetic Programming (GP) [14]. wherein tree-based structures develop, which determines the relationship between the assessment and reaction between the different variables. GEP is commonly a genotype as well as phenotype genetic algorithm that matures computer programs to discover the solution of the applicant problem constructed on Darwin's theory of reproduction, crossover, and mutation. The main performers in GEP are the chromosomes and the Expression Trees (ETS).GEP assimilates both the simple and linear chromosomes of stable length analogous to the ones castoff in genetic algorithms [15]. The novel concept behind the linear chromosomes and the Expression Trees helps GEP to considerably overhaul prevailing adaptive algorithms. Therefore, GEP algorithms having new prospects for solving more complicated and logical problems [16].

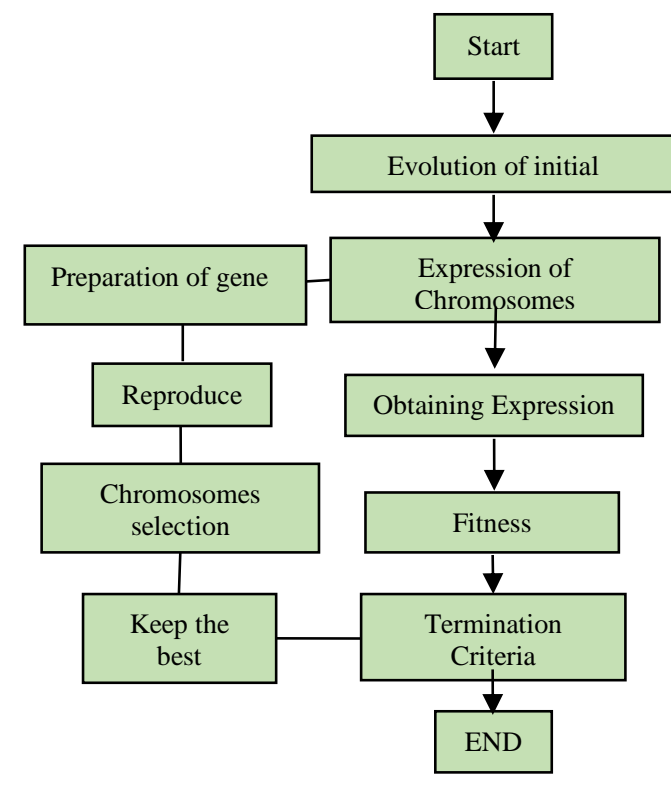

Fig. 1. Flow chart of GEP algorithms

\section{GEP Architecture.}

The most important factors of GEP are the function set, terminal set, fitness function, control parameters, and stop condition which requires to be preset while using the GEP model to resolve a particular problem [17,18]. The characters are encoded as linear strings of stationary size (genome) in GEP, which are conveyed later as non-linear individuals with altered size and shapes; and are well-known as Expression Trees as shown in Fig 3. 


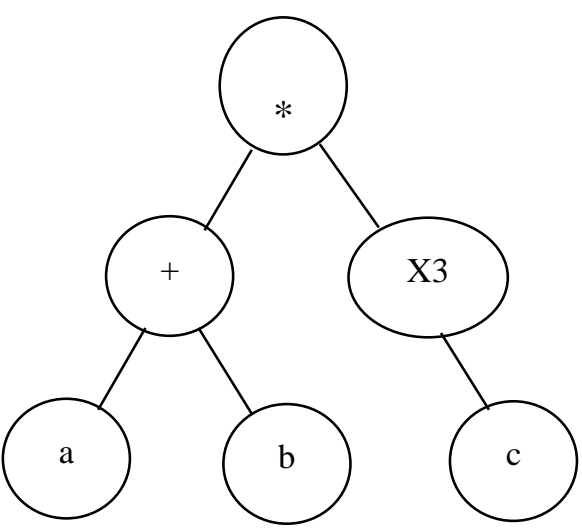

Fig. 2. Example of GEP expression tree (ET).

Fig 2 comprises in GEP algorithms and the Equation develops as $(a+b) * c^{3}$. GEP employs a head-tail method. Each GEP gene is composed of a head and a tail. The head may have jointly function and terminal symbols, where the tail may hold only terminal symbols [13]. The size of the tail section is:

$\mathrm{T}=(\mathrm{a}-1)^{*} \mathrm{~h}+1$, Where, $\mathrm{h}$ is the size of the head section and $\mathrm{a}$ is the maximum arity In this study, the basic arithmetic operators $\left(+,-,{ }^{*}, /\right)$ and diverse mathematical functions (Pow, Sqrt, Exp, Ln, Log, 1/x, $x^{2}, x^{3}$, Cube root, Sin, $\mathrm{Cos})$ were employed to develop the anticipated GEP model. Bigger population size takings lengthier for an iteration run. The program was run until there was no substantial enhancement in the performance of the models. The recent study was assumed to accomplish an unambiguous relationship between different variables (Pipe Diameter, Liquid Density, Solid Density, Liquid Viscosity, Volume Fraction, and Solid Concentration) with the verdict variables (Pressure drop). The different parameters of the GEP model are shown in table. 2 and the function used is shown in table 3.

Table 2. Parameters of the GEP model.

\begin{tabular}{cc}
\hline Mathematical functions & $\begin{array}{c}+, \ldots, *, /, \text { Pow, Sqrt, Exp, } \\
\operatorname{Ln}(\mathrm{x}), \log , \operatorname{Inv}(1 / \mathrm{x}), \mathrm{x}^{2}, \mathrm{x}^{3}, \\
\operatorname{Cube} \operatorname{root}(3 \mathrm{Rt}), \operatorname{Sin}, \mathrm{Cos}\end{array}$ \\
\hline Number of Chromosomes & $30,60,90,120,150$ \\
Number of genes & $2,3,4,5,6$ \\
Head size & $5,10,15,20$ \\
Linking function & addition \\
Mutation rate & 0.044 \\
Inverse rate & 0.1 \\
One-point recombination rate & 0.3 \\
Two-point recombination rate & 0.3 \\
Gene recombination rate & 0.1 \\
Gene transportation rate & 0.1 \\
\hline
\end{tabular}

Table 3. Definition of the functions used in the GEP model.

\begin{tabular}{|c|c|c|c|c|}
\hline Weight & Function & Representation & Arity & Definition \\
\hline 4 & addition & + & 2 & $(\mathrm{X}+\mathrm{Y})$ \\
\hline 4 & subtraction & - & 2 & $(\mathrm{X}-\mathrm{Y})$ \\
\hline 4 & multiplication & $*$ & 2 & $\left(X^{*} Y\right)$ \\
\hline 1 & division & / & 2 & $(\mathrm{X} / \mathrm{Y})$ \\
\hline 1 & Power & pow & 2 & $\operatorname{Pow}(X, Y)$ \\
\hline 1 & Square root & sqrt & 1 & $\operatorname{Sqrt}(X)$ \\
\hline 1 & exponential & $\exp$ & 1 & $\operatorname{Exp}(x)$ \\
\hline 1 & Natural logarithm & $\ln$ & 1 & $\operatorname{Ln}(x)$ \\
\hline 1 & Logarithm of base 10 & $\log$ & 1 & $\log (x)$ \\
\hline 1 & inverse & inv & 1 & $1 / \mathrm{x}$ \\
\hline 1 & $\mathrm{X}$ to the power of 2 & $\mathrm{X} 2$ & 1 & $X^{\wedge} 2$ \\
\hline 1 & $\mathrm{X}$ to the power of 3 & X3 & 1 & $\mathrm{X}^{\wedge} 3$ \\
\hline 1 & Cube root & $3 \mathrm{Rt}$ & 1 & $X^{\wedge}(1 / 3)$ \\
\hline 1 & Sine & Sin & 1 & $\operatorname{Sin}(x)$ \\
\hline 1 & Cosine & Cos & 1 & $\operatorname{Cos}(x)$ \\
\hline
\end{tabular}




\section{Artificial Neural network}

ANN is an effective tool generally used to predict accurately using biological neurons,[19]which was presented by McCulloch and Pitts 1943[20]. An ANN methodology is used to solve the most complicated problems to minimize complexity. Its application is not limited to basic engineering problems rather it resolves different problems such as recognition, nonlinear modeling, classification, association, and control. The most important characteristics of ANN can be expressed by its architecture and the method of determining the activation function [21].

The present study aims to predict the pressure drop by ANN as well as GEP and to compare the results of both soft tools. The data used in this study are used to find pressure drops in a pipeline system having various pipe diameters. This study uses two-layered ANN architecture as shown in fig 3 with 10 neurons. The optimal number of hidden layers and the number of neurons relative to each hidden layer is obtained by the performance attained by the ANN control system and the number of epochs during training.

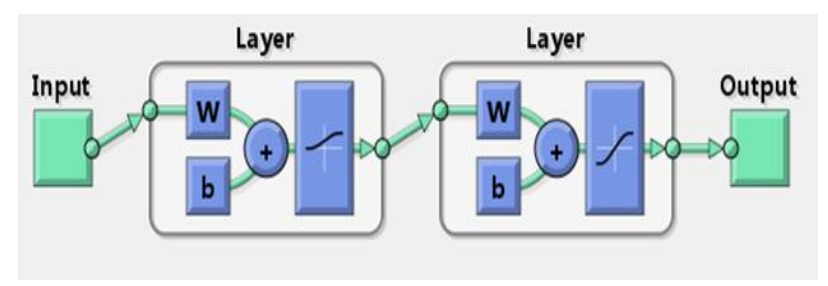

Fig. 3. ANN architecture.

The ANN model has been developed by using MATLAB $n-n$ tool for the prediction of Pressure drop. $70 \%$ of the data are being used for training the network and the remaining $30 \%$ data is used for validation. ANN predict pressure drop with a mean square error of 1.3. The various adaptive features used in this model are shown in table 4 .

Table 4 Different ANN features used in the model

\begin{tabular}{cc}
\hline Features & Function \\
\hline Training function & TRAINSCG \\
Adaption learning function & LEARNGDM \\
Performance function & MSE \\
No of Hidden layer & 2 \\
Transfer function & LOGSIG \\
Network Type & Feedforward back prop \\
\hline
\end{tabular}

\section{E. Statistical analysis of the Model}

The correlation coefficient (R2-value), absolute error(AE), relative error (RE), mean squared error (MSE)a root mean square error (RMSE) were cast-off for assessing and equaling the prediction performance of ANN and GEP models.

$$
\begin{gathered}
A E=\left|f_{\text {true }}-f_{\text {predicted }}\right| \\
R E=\left(\frac{\left|f_{\text {true }}-f_{\text {predicted }}\right|}{f_{\text {true }}}\right) 100 \\
M S E=\frac{1}{n} \sum_{i=1}^{n}\left(f_{\text {true }}-f_{\text {predicted }}\right)^{2} \\
R^{2}=1-\left[\frac{\sum_{i=1}^{n}\left(t_{i}-o_{i}\right)^{2}}{\sum_{i=1}^{n}\left(o_{i}\right)^{2}}\right] \\
M A P E=\frac{1}{n} \sum_{i=1}^{n}\left(\frac{t_{i}-o_{i}}{t_{i}}\right) \times 100
\end{gathered}
$$

Where $f_{\text {true }}$ is the true value of pressure drop, $p_{\text {redicted }}$ the predicted pressure drop from different methods and $\mathrm{n}$ the number of predicted values" is the experimental output and ' $\mathrm{o}$ ' is the predicted output value. 


\section{Results and Discussion}

\section{A.GEP algorithms Results}

The most important analogy of GEP is that the problem function needs to be well defined. Then the program hunts for a solution in a problem sovereign manner. Hence all functions which are used in this GEP model are defined as per the model structure. GEP comprises a parse tree generator that spontaneously changes the native Karva code of our logic circuits into diagram demonstrations or expression trees (Fig. 4 \& Fig. 5), permitting a faster and more complete indulgence of their Boolean structure. The best-generated formula by GEP model to find out the pressure drop is represented the Eq.1

$$
\begin{gathered}
\text { Pressure drop }=\ln \left(\left(d_{1}-\left(c_{1}+c_{4}\right)\right)-\left(\ln d_{4}\right)^{\frac{1}{3}}\right)+ \\
\left(\left(c_{4}+d_{7}\right) \times\left(d_{6} \times c_{7}\right)\right)^{\frac{1}{3}} \times\left(\left(d_{1} \times d_{0}\right)-d_{4}\right)+\left(c_{3}-d_{3}\right)+d_{6}+\frac{1}{d_{4}}-c_{3}+d_{1}+\left(d_{3} \times\left(d_{3}-d_{5}^{2}\right) \times d_{4}\right)^{\frac{1}{3}}
\end{gathered}
$$

The different statistical analyses of the data used for ANN and GEP evaluated to find out absolute error, Relative error, Mean squared error, correlation coefficient, and root means square error. The comparison of GEP model predictions with the ANN model for pressure drop in the different pipeline system is indicated in Table.5.

Table 5. Statistical Analysis of the Results

\begin{tabular}{cc}
\hline \multirow{2}{*}{ Variable } & Pressure drop \\
\cline { 2 - 2 } & GEP \\
\hline $\mathrm{AE}$ & 8.993 \\
$\mathrm{RE}$ & 6.566 \\
$\mathrm{MSE}$ & 2310.226906 \\
$\mathrm{R}^{2}$ & 0.999153373 \\
$\mathrm{MAPE}$ & 6.566600297 \\
\hline
\end{tabular}

The results obtained by both the model ANN and GEP are statically analyzed to find out Absolute error, Relative error, mean squared error, correlation coefficient and mean absolute percentage error in this study. The GEP model predicts with more accuracy of $\mathrm{R}$ square value 0.99153373 which is much closer to 1 as compared to the ANN model. Although the absolute error percentage is also found to be less in GEP as compared to the ANN model. The main objective of the study was to predict the pressure drop more accurately by soft computing methods. The different input parameters effectively describe the output variable to find-out pressure drop. Moreover, ANN is an effective tool used for predicting any type of engineering problems to solve complicated problems.

Sub- ET 1

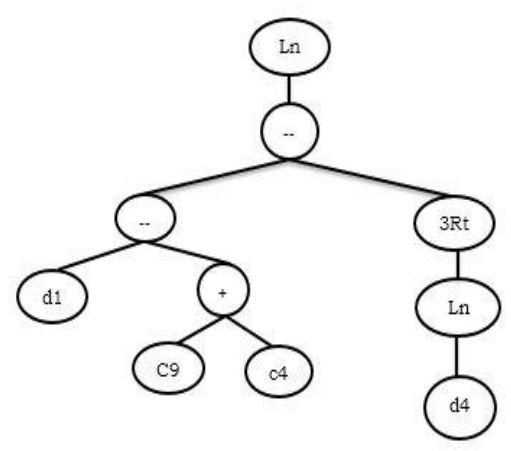

Sub-ET 2

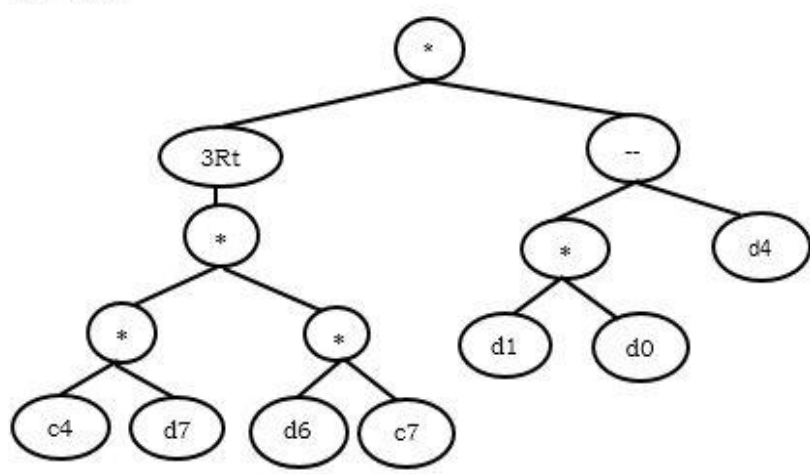


Sub-ET 3

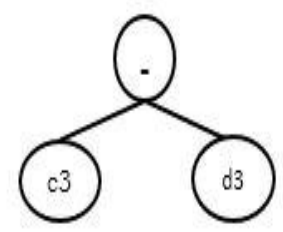

Fig.4.Tree Diagram Expression for the predicted pressure drop from sub ET(1-4)

Sub-ET 5

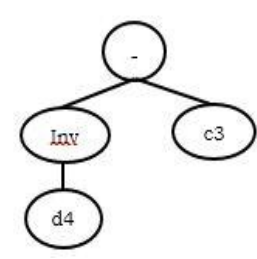

Sub-ET 7

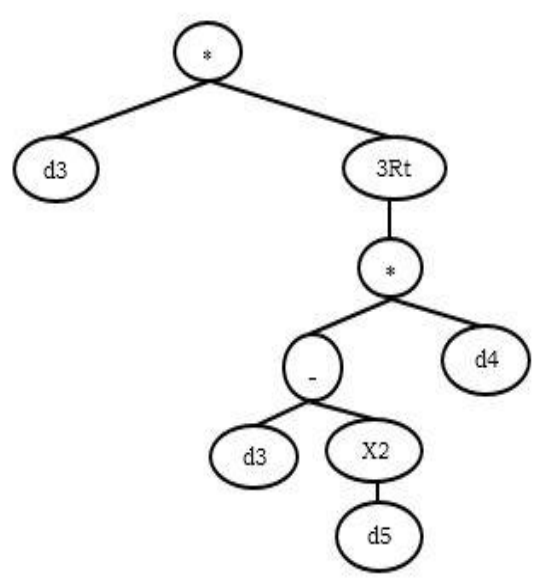

Sub-ET 8

Sub-ET 9

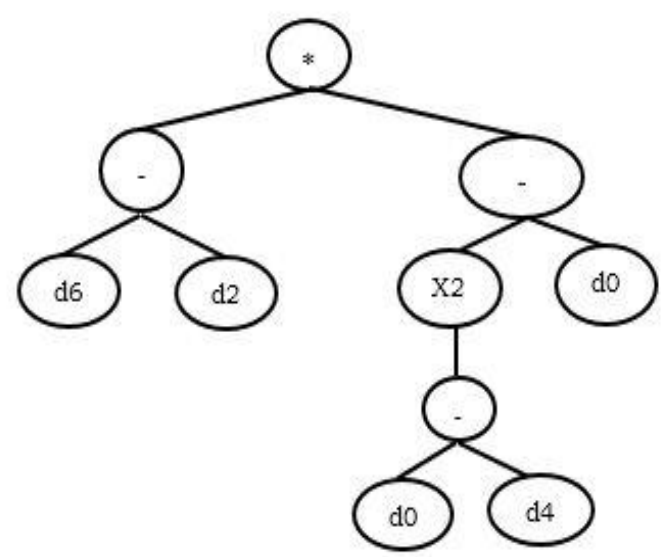

Sub-ET 10

\section{Sub-ET 4}

d6
Sub-ET 6
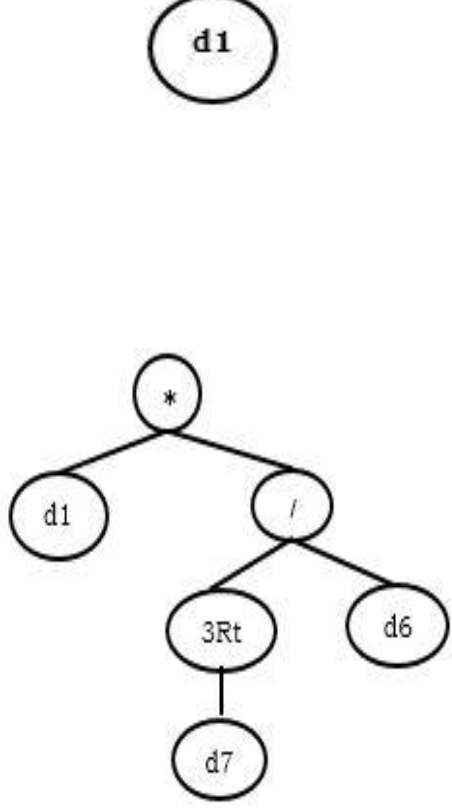

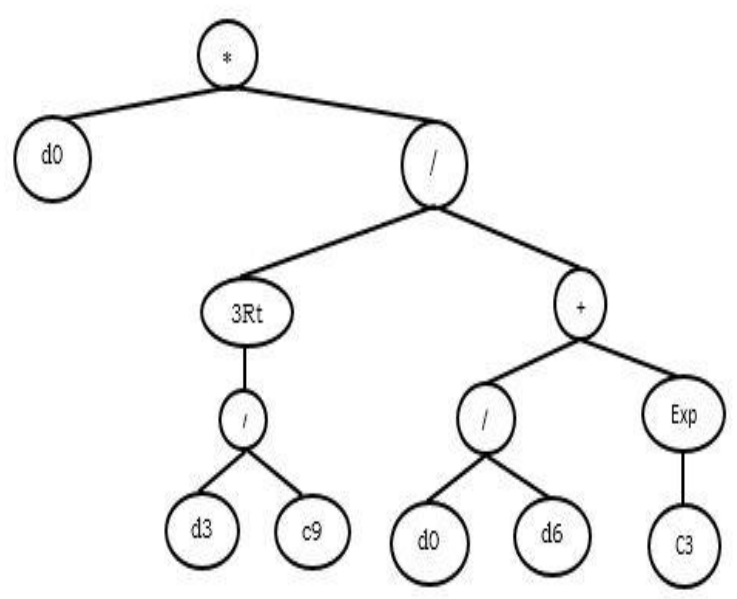

Fig.5. Tree Diagram Expression for the predicted pressure drop from sub ET(5-10) 
The fig. 6 shows comparative analysis between the target data and predicted data in the analysis of the GEP model. The predicted data for the output variable pressure drop throughout the pipeline system has been studied statistically to discretize the percentage of deviation of predicted data from the experimental values. The input data shows a clear agreement to follow the trend line.Fig.7 shows the deviation of the data from the predicted trend line in the GEP model. Moreover, the output data has compared with the predicted data of the ANN model to find out the variation of both the model is shown in fig.8 and 9.

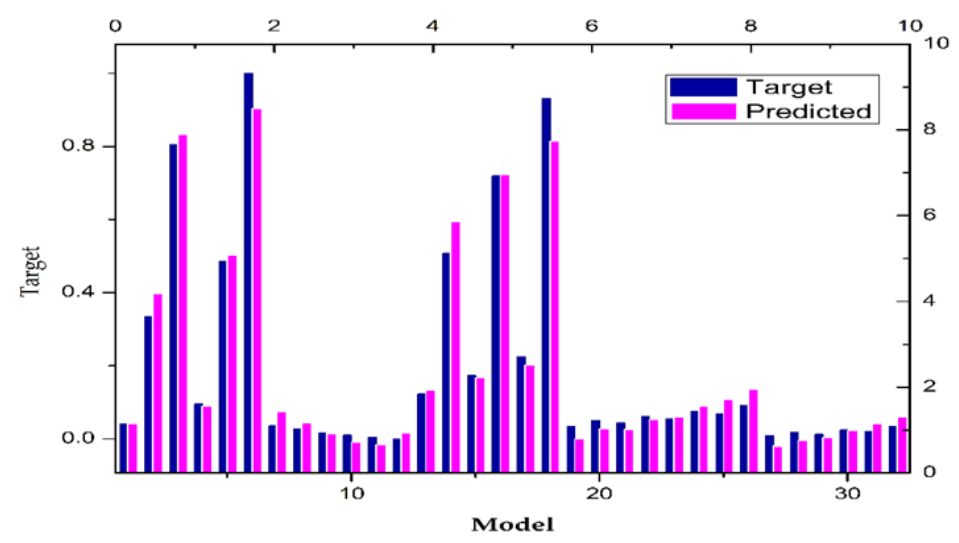

Fig. 6. Comparisons between the target data and predicted data.

Table 6: Results obtained by ANN Model.

\begin{tabular}{cc}
\hline \multirow{2}{*}{ Variable } & Pressure drop \\
\cline { 2 - 2 } & ANN \\
\hline RE & 15.566 \\
MSE & 9.233 \\
$\mathbf{R}^{2}$ & 30621.45078 \\
MAPE & 0.988267825 \\
\end{tabular}

\section{B. Artificial Neural Network Results.}

Artificial Neural Networks (ANNs) are soft computing methods and are being inspired by the neural architecture of the human brain. ANNs models are generally developed to resemble the learning methods of biological neural systems of the human brain. The pressure drop parameters are predicted with an absolute error of $15.566 \%$. The relative error is found to be 9.233 and the mean absolute percentage error as 23.294. The results of ANN models are listed in table 6.

The results obtained by ANN architecture and its validation are shown in the figure
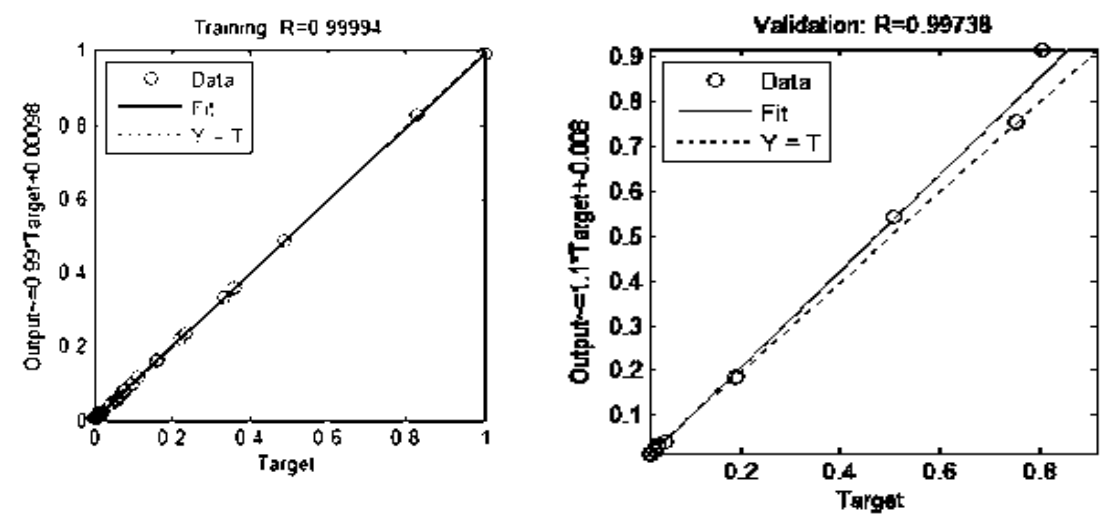

Fig.7.ANN training and Test data 

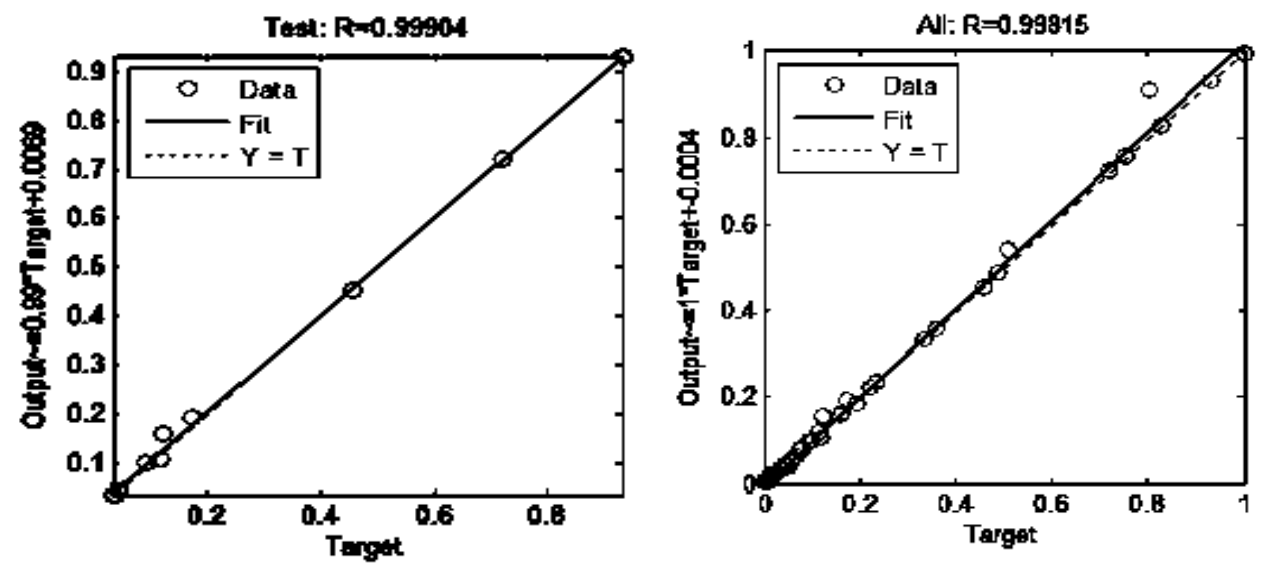

Fig.8. ANN model validation.

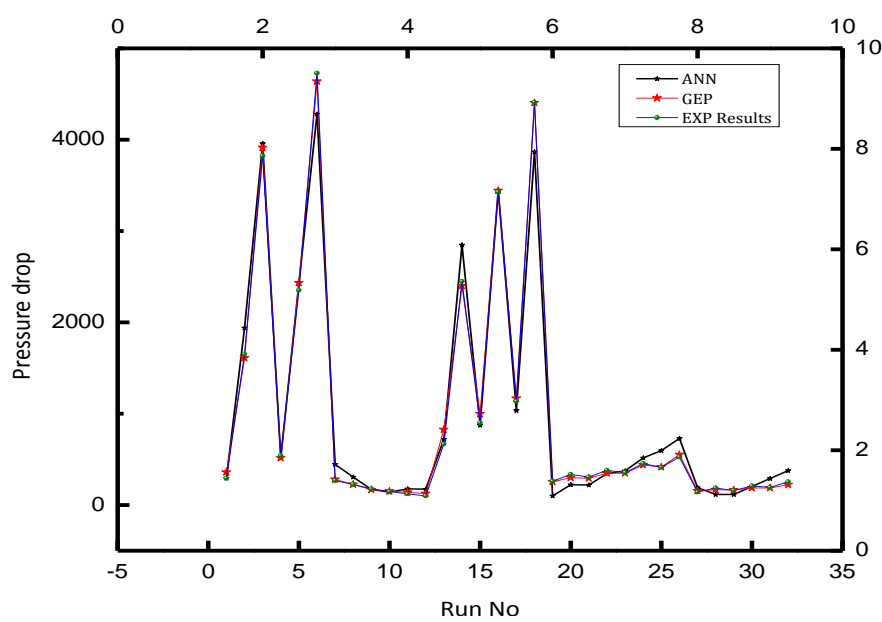

Fig.9.Comparison of ANN, GEP with Experimental data

\section{Conclusion}

The present study tries to predict the pressure drop in different pipeline setup using previous literature using ANN and GEP. The predicted results by both the model were compared. The numerical results of both the model have been evaluated in the graph and also analyses the error percentage to compare the best model. The ANN predicted data has been compared with the experimental data the error percentage was found to be ranging from 2.2 to 2.4 whereas the error percentage in the case of the GEP model was found to be ranging from 1.2 to 1.35 . Gene expression model predicts with an accuracy of mean R-Square 0.982288 and a correlation coefficient of 0.991104 for the pressure drop. The GEP model was found to be the best model in terms of predicting pressure drop with a correlation for predicting pressure drop given by the equation 6. Although the other parameter related to the pipeline system may also be evaluated to have high accuracy.

The GEP model performance in terms of prediction is found to be more superior as compared to the ANN model. The main advantage of using the GEP model is that it develops a correlation concerning the input variables and thus predict the output variable with a formula. This provides an explicit area for parametric co-relationship among different input variables and resolves the optimal solution.

\section{Acknowledgement}

Authors are exceptionally appreciative of NIT Agartala for providing Experimental set up to complete the experiment successfully and Special thanks to Binayak Sen and Syed Abou Iltaf Houssen for his extreme support in the field of ANN and GEP simulation. 


\section{References}

[1] Ghanta, K.C, and Purohit, N.K, (1999). Pressure drop prediction in hydraulic transport of bi - dispersed particles of coal and copper ore in the pipeline, The Canadian Journal of Chemical Engineering, 77(1), pp. 127-131.

[2] Gillies, R.G. and Shook, C.A., (2000). Modeling high concentration settling slurry flows. The Canadian Journal of Chemical Engineering, 78 (4), pp. 709-716

[3] S.K. Lahiri, K.C. Ghanta,( 2008) Development of an artificial neural network correlation for prediction of the hold-up of slurry transport in pipelines, Chemical Engineering Science, 63, 6, 1497.

[4] K.C. Wilson, R.S. Sanders, R.G. Gillies, C.A. Shook,(2010) Verification of the near-wall model for slurry flow, Powder Technology, 197, 3, 247.

[5] Manoj Kumar Gopaliya, D. R. Kaushal,( 2015) Analysis of Effect of Grain Size on Various Parameters of Slurry Flow through Pipeline Using CFD, Particulate Science, and Technology, 33, 4, 369.

[6] Wasp EJ, Aude TC. (1970)Deposition velocities, transition velocities, and spatial distribution of solids in slurry pipelines, In Presented at the 1st International British Hydromechanics Research Association Hydraulic Transport of Solids in Pipes Conference, War Wickshire Univ, Coventry, England, Sept 1-4, 1970, (No. H4 Proceeding).

[7] Bandyopadhyay JK, Annamalai S, Gauri KL. (1996) Application of artificial neural networks in modeling limestone-SO2 reaction, AIChE journal. Aug 1; 42 (8):2295-302.

[8] Bowen WR, Jones MG, Yousef HN (1998). Prediction of the rate of cross-flow membrane ultra-filtration of colloids: A neural network approach. Chemical Engineering Science. Nov 1; 53(22):3793-802.

[9] Roy S, Ghosh A, Das AK, Banerjee R.( 2014) A comparative study of GEP and an ANN strategy to model engine performance and emission characteristics of a CRDI assisted single-cylinder diesel engine under CNG dual-fuel operation, Journal of Natural Gas Science and Engineering, Nov 30; 21: 814-28.

[10] Dey, P., Sarkar, A., \& Das, A. K. (2015). Prediction of unsteady mixed convection over a circular cylinder in the presence of nanofluid-A comparative study of ANN and GEP. Journal of Naval Architecture and Marine Engineering, 12(1), 57-71.

[11] Dey, P., Sarkar, A., \& Das, A. K. (2017). Capability to predict the steady and unsteady reduced aerodynamic forces on a square cylinder by ANN and GEP. Neural Computing and Applications, 28(8), 1933-1945.

[12] S.K. Lahiri, K.C. Ghanta. (2008)Development of an artificial neural network correlation for prediction of the hold-up of slurry transport in pipelines, Chemical Engineering Science 63 1497- 1509.

[13] Ferreira C. (2001) Gene expression programming: a new adaptive algorithm for solving problems. Complex Syst; 13:87-129.

[14] Koza JR. (1995) Survey of genetic algorithms and genetic programming. In: WESCON/ ' 95 conference record 'microelectronics communications technology producing quality products mobile and portable power emerging technologies'; p. 589.

[15] Guven A. (2009) Linear genetic programming for time-series modeling of daily flow rate. J Earth Syst Sci; 118:137-46.

[16] Wan Tang, Limei Peng, Ximin Yang, Xia Xie, Yang Cao,( 2010), GEP-based Framework for Immune- Inspired Intrusion Detection, KSII Transactions on Internet and Information Systems Vol. 4, No.6, December 23, doi.10.3837/tiis.2010.12.017.

[17] Xinyu Li, Ping Jiang, Liping Zhang. (2014)Prediction of surface roughness in end milling with gene expression programming. In Proceedings of the 41st International Conference on Computers \& Industrial Engineering.

[18] A.R. Fallahpour, A.R. Moghassem. (2013) Yarn Strength Modelling Using Adaptive Neuro-Fuzzy Inference System (ANFIS) and Gene Expression Programming (GEP). Journal of Engineered Fibers and Fabrics Volume 8, Issue 4.

[19] S. Haykins, (1994) Neural Networks: A Comprehensive Foundation, MacMillan, New York.

[20] W.S. McCulloch, W. Pitts,( (1943)) A logical calculus of the ideas immanent in nervous activity, Bull. Math. Biophys. 5, 115133.

[21] L. Fausett,( 1994.) Fundamentals of Neural Networks, Prentice-Hall, Englewood Cliffs, N.J,

\section{Authors' Profiles}



Rajesh Chakraborty is a Ph.D. scholar at the Department of Production Engineering. National Institute of Technology, Agartala, Tripura, India. He received a B.Tech degree from Tripura Institute of Technology, Narsingarh, Tripura in Mechanical Engineering and M.Tech Degree from National Institute of Technology, Agartala, Tripura, India in 2011 and 2013, respectively. His research interests include Multiphase flow modeling and simulation, MCDM Techniques, Neural computing, Pipe flow analysis.

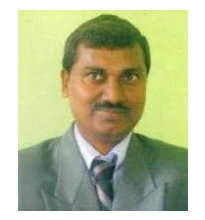

Dr. Uttam Kumar Mandal Assistant Professor and HOD at the Department of Production Engineering. National Institute of Technology, Agartala, Tripura, India. He received his B.Tech degree from Jadavpur University, in Mechanical Engineering and M.Tech Degree and also received a Ph.D. from Jadavpur University, West Bengal, India.

Dr. Mandal has published many papers in different National and International journals and also reviewers in many journals. His areas of interest include Manufacturing Technology, Multi-Criteria Decision Making (MCDM), Case-Based Reasoning (CBR), and Advanced Fluidics. He is a member of the Institute of Engineers India (IEI). 


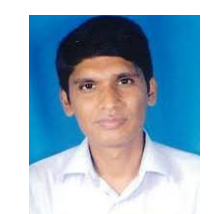

Dr. Rabindra Nath Barman Assistant Professor at the Department of Mechanical Engineering National Institute of Technology, Durgapur, West Bengal, India. He received a B.Tech degree from Jadavpur University, in Mechanical Engineering and M.Tech Degree and also received a Ph.D. from Jadavpur University, West Bengal, India in the year 2003, 2005 and 2012 respectively.

Dr. Barman was the former Assistant professor in NIT Agartala (2010-2014) and joined NIT Durgapur in 2014 to till date. He has published papers in many National and International journals. He has published book (2019) Numerical Investigation of $\mathrm{Cu}-\mathrm{H} 2 \mathrm{O}$ nano-fluid in a Differentially Heated Square Cavity with Conducting Square Cylinder Placed at Arbitrary Locations. Innovative Design, Analysis and Development Practices in Aerospace and Automotive Engineering (I-DAD 2018). Lecture Notes in Mechanical Engineering. Springer, Singapore. His areas of interest include Fluid Mechanics, Heat Transfer, Computational Fluid Dynamics (CFD), Modeling and Simulation. He is also a member of the American Society of Mechanical Engineers (ASME) and Institute of Engineers India (IEI).

How to cite this paper: Rajesh Chakraborty, Uttam Kumar Mandal, Rabindra Nath Barman, " A Comparative Study of ANN and GEP Model to Predict the Pressure Drop in the Water Transportation System", International Journal of Information Engineering and Electronic Business(IJIEEB), Vol.12, No.5, pp. 47-57, 2020. DOI: 10.5815/ijieeb.2020.05.05 